\title{
The right choice of antihypertensives protects primary human hepatocytes from ethanol- and recombinant human TGF- $\beta_{1}$-induced cellular damage
}

\author{
This article was published in the following Dove Press journal: \\ Hepatic Medicine: Evidence and Research \\ 18 March 2013 \\ Number of times this article has been viewed
}

\author{
Sabrina Ehnert ${ }^{1}$ \\ Teresa Lukoschek ${ }^{2}$ \\ Anastasia Bachmann² \\ Juan J Martínez Sánchez' \\ Georg Damm ${ }^{3}$ \\ Natascha C Nussler ${ }^{4}$ \\ Stefan Pscherer ${ }^{5}$ \\ Ulrich Stöckle' \\ Steven Dooley ${ }^{2}$ \\ Sebastian Mueller ${ }^{6}$ \\ Andreas K Nussler' \\ 'Eberhard Karls Universität Tübingen, \\ BG Trauma Center, Tübingen, \\ Germany; ${ }^{2}$ Mol Hepatology - Alcohol \\ Associated Diseases, Department \\ of Medicine II, Medical Faculty, \\ Mannheim, Germany; ${ }^{3}$ Department of \\ General,Visceral, and Transplantation \\ Surgery, Charité University Medicine, \\ Berlin, Germany; ${ }^{4}$ Clinic for General, \\ Visceral, Endocrine Surgery and \\ Coloproctology, Clinic Neuperlach, \\ Städtisches Klinikum München $\mathrm{GmbH}$, \\ Munich, Germany; ${ }^{5}$ Department of \\ Diabetology, Klinikum Traunstein, \\ Kliniken Südostbayern AG, Traunstein, \\ Germany; ${ }^{6}$ Department of Medicine, \\ Salem Medical Center, Ruprecht- \\ Karls-Universität, Heidelberg, \\ Germany
}

Correspondence: Andreas Nüssler Eberhard Karls Universität Tübingen, BG Trauma Center, Schnarrenbergstr 95, 72076 Tübingen, Germany

Tel +49 707I 606 I065

Fax +49 707| 606 I978

Email andreas.nuessler@googlemail.com
Background: Patients with alcoholic liver disease (ALD) often suffer from high blood pressure and rely on antihypertensive treatment. Certain antihypertensives may influence progression of chronic liver disease. Therefore, the aim of this study is to investigate the impact of the commonly used antihypertensives amlodipine, captopril, furosemide, metoprolol, propranolol, and spironolactone on alcohol-induced damage toward human hepatocytes (hHeps).

Methods: hHeps were isolated by collagenase perfusion. Reactive oxygen species (ROS) were measured by fluorescence-based assays. Cellular damage was determined by lactatedehydrogenase (LDH)-leakage. Expression analysis was performed by reverse-transcription polymerase chain reaction and Western blot. Transforming growth factor (TGF)- $\beta$ signaling was investigated by a Smad3/4-responsive luciferase-reporter assay.

Results: Ethanol and TGF- $\beta_{1}$ rapidly increased ROS in hHeps, causing a release of $40 \%-60 \%$ of total LDH after 72 hours. All antihypertensives dose dependently reduced ethanol-mediated oxidative stress and cellular damage. Similar results were observed for TGF- $\beta_{1}$-dependent damage, except for furosemide, which had no effect. As a common mechanism, all antihypertensives increased heme-oxygenase-1 (HO-1) expression, and inhibition of HO-1 activity reversed the protective effect of the drugs. Interestingly, Smad3/4 signaling was reduced by all compounds except furosemide, which even enhanced this profibrotic signaling. This effect was mediated by expressional changes of Smad3 and/or Smad4.

Conclusions: Our results suggest that antihypertensives may both positively and negatively influence chronic liver disease progression. Therefore, we propose that in future patients with ALD and high blood pressure, they could benefit from an adjusted antihypertensive therapy with additional antifibrotic effects.

Keywords: primary human hepatocytes, alcoholic liver disease, ethanol, TGF- $\beta_{1}$, antihypertensives

\section{Introduction}

Alcoholic liver disease (ALD) is one of the leading causes of death worldwide. It progresses from steatohepatitis to clinical manifestations, such as fibrosis, cirrhosis, and hepatocellular carcinoma. Ethanol consumption leads to production of reactive oxygen species (ROS), eg, hydroxyl-ethyl free radicals, hydroxyl radicals, and superoxide anions, causing an accumulation of reactive compounds and lipid peroxidation in ALD patients. ${ }^{1}$ ROS are produced during ethanol metabolism by both enzymes and, more selectively, through the microsomal ethanol oxidative system with its main 
component cytochrome P450 (CYP)2E1. ${ }^{2}$ In a previous study, we showed that ethanol and recombinant human transforming growth factor (rhTGF)- $\beta_{1}$ induce oxidative stress in human hepatocytes (hHeps) with a peak of ROS levels and cellular glutathione depletion after 4 hours. Continuous exposure to both substances results in apoptotic cell death with 40\%-60\% of total lactate-dehydrogenase (LDH) released into the culture medium after 72 hours. ${ }^{3}$ In ALD patients, upregulated CYP2E1 expression goes along with iron mobilization ${ }^{4}$ and reduction of antioxidant enzymes and chemicals, particularly mitochondrial and cytosolic glutathione. ${ }^{3}$ This causes a disbalance between cell ROS production and cellular enzymatic and nonenzymatic defense mechanisms. Generated ROS then react with and in turn damage complex cellular molecules, eg, lipids, proteins, DNA, mitochondria, and cell membranes. ${ }^{5}$ Such repeated damage causes liver cells to produce excessive extracellular matrix, which eventually prevents organ regeneration. Furthermore, ethanol affects the immune system by altering cytokine production. ${ }^{6}$ This is of particular importance as hHeps exposed to ethanol are more sensitive toward damage from TGF- $\beta_{1},{ }^{7}$ which enhances oxidative stress on hHeps by upregulating nicotinamide adenine dinucleotide phosphate-oxidases (NOX). ${ }^{8}$ Furthermore, TGF- $\beta$ signal transduction in this and other liver cell types is critically required for liver disease progression.

Reducing oxidative stress by upregulating the endogenous antioxidative enzyme heme-oxygenase-1 (HO-1) with cobalt protoporphyrin protected $\mathrm{hHeps}$ from ethanol-induced cytotoxicity. ${ }^{9}$ However, most HO-1 inducers, including cobalt protoporphyrin and hemin, show toxic side effects. Just recently we showed a protective effect of the naturally occurring polyphenol quercetin against ethanol-induced cellular damage in hHeps being dependent on HO- $1 .{ }^{10,11}$ Similar effects were shown for the structurally related calcium channel blockers nifedipine and verapamil, ${ }^{3}$ both formerly used as standard drugs to regulate blood pressure. Therapeutic targets for constantly controlling high blood pressure have continued to evolve, depending on the patients' disease pathology, age, and other factors, and corresponding drugs have been developed. In addition to the aforementioned calcium channel blockers (calcium antagonists), these include diuretics, angiotensin-converting enzyme (ACE)-inhibitors, aldosterone antagonists, renin inhibitors, as well as adrenergic receptor agonists and antagonists ( $\alpha$ - and $\beta$-blockers). There are hints that certain antihypertensives influence fibrosis in vivo, as reported for myocardial and renal fibrosis. ${ }^{12,13}$ There are reports that amlodipine and captopril may also be effective against lung and liver fibrosis as well as keloid formation. ${ }^{14-17}$
However, the available data are contradictory, eg, the ACEinhibitor captopril may reduce as well as enhance left ventricular fibrosis in rats. ${ }^{12,13}$ As patients with ALD often suffer from high blood pressure, they rely on an antihypertensive treatment. Therefore, the aim of this study was to investigate the influence of the commonly used antihypertensives, amlodipine (calcium channel blocker), captopril (ACE-inhibitor), furosemide (diuretic), metoprolol ( $\beta_{1}$-blocker), propranolol ( $\beta_{1} / \beta_{2}$-blocker), and spironolactone (aldosterone antagonist) on alcohol- and TGF- $\beta_{1}$-induced damage in hHeps.

\section{Materials and methods}

rhTGF- $\beta_{1}$ was purchased from Peprotech (London, UK). Chemicals, cell culture medium, and supplements, if not stated differently, were purchased from Sigma (Munich, Germany). Antibodies were purchased from Santa Cruz Biotechnology (Heidelberg, Germany).

\section{Isolation of primary hHeps}

Human liver tissue was obtained according to institutional guidelines ("Klinikum rechts der Isar," Technical University Munich, Munich, Germany) from liver resections of tumor patients with primary or secondary liver tumors. Written informed consent was obtained from all patients. hHeps were isolated by a two-step collagenase perfusion technique followed by Percoll gradient centrifugation. ${ }^{18}$ hHeps viability (trypan blue exclusion) was consistently above $85 \%$. hHeps were cultured on collagencoated culture plates in Williams' Medium E $(10 \%$ fetal calf serum, $1 \%$ nonessential amino acids, $1 \mathrm{mM}$ sodium pyruvate, 32 IE/L insulin (Actrapid ${ }^{\circledR}$; Novo Nordisk Pharma GmbH, Mainz, Germany), $15 \mathrm{mM}$ HEPES, $0.8 \mu \mathrm{g} / \mathrm{mL}$ hydrocortisone, $100 \mathrm{U} / \mathrm{mL}$ penicillin, and $100 \mathrm{mg} / \mathrm{mL}$ streptomycin). Prior to all experiments, hHeps were serum starved overnight.

\section{ROS measurement}

hHeps, stimulated for 4 hours with $100 \mathrm{mM}$ ethanol and/or $5 \mathrm{ng} / \mathrm{mL}$ rhTGF- $\beta_{1}$ in the presence or absence of antihypertensives as indicated, were washed twice with Dulbecco's phosphate-buffered saline. Cells were then incubated with $10 \mu \mathrm{M} \mathrm{2} 2^{\prime}, 7^{\prime}$-dichlorfluorescein-diacetate (ROS) for $30 \mathrm{~min}$ utes at $37^{\circ} \mathrm{C}$ in serum-free culture medium. The amount of ROS is in concordance with the fluorescence measured at an excitation/emission of 485/520 nm. ${ }^{3}$ Basal ROS levels were determined in untreated cells.

\section{Cellular damage}

LDH activity in the culture supernatant was determined by the LDH test kit from Fa Hitado (Möhnesee, Germany) 
according to the manufacturer's protocol. Results are given as percentage of total LDH released. A $100 \%$ LDH release was determined by cell lysis with $0.1 \%$ Triton X-100. Untreated cells were used to determine the basal cell death over the culture period.

\section{Conventional reverse-transcriptase polymerase chain reaction}

Total cellular RNA was isolated with Trifast (Peqlab, Erlangen, Germany) according to the manufacturer's protocol. First-strand cDNA was synthesized from $1 \mu \mathrm{g}$ RNA according to the manufacturer's instructions by using the First Strand cDNA Synthesis kit from Fermentas (St Leon-Rot, Germany). Primer data are summarized in Table 1. Products resolved by gel electrophoresis were visualized with ethidiumbromide.

\section{Western blot}

hHeps were lysed in ice-cold lysis buffer (50 mM TRIS [Trisaminomethane], $250 \mathrm{mM} \mathrm{NaCl}, 2 \%$ Nonidet P40, $2.5 \mathrm{mM}$ EDTA [Ethylenediaminetetraacetic acid], $0.1 \%$ sodium dodecyl sulfate [SDS], $0.5 \%$ deoxycholic acid, complete protease inhibitor, $1.0 \%$ phosphatase inhibitor, $\mathrm{pH}$ 7.2). Protein concentration was determined by micro-Lowry. Thirty micrograms total protein was separated by SDS-polyacrylamide gel electrophoresis and transferred to nitrocellulose membranes (Roth, Karlsruhe, Germany). After overnight incubation with primary antibodies (1:1000 in Tris-buffered saline with Triton-X-100) at $4{ }^{\circ} \mathrm{C}$, membranes were incubated with the corresponding horseradish peroxidase-labeled secondary antibodies for 2 hours at room temperature. Chemiluminescent signals were detected with X-ray film.

\section{Statistics}

Results are expressed as mean \pm standard error of the mean of at least three independent experiments $(\mathrm{N} \geq 3)$ measured in triplicate or more $(n \geq 3)$. Densitometric analysis was performed using ImageJ software (National Institutes of Health, Bethesda, MD, USA). Data sets were compared by one-way analysis of variance followed by Bonferroni's multiple comparison test (GraphPad Prism software; GraphPad Software, Inc, La Jolla, CA, USA). $P<0.05$ was taken as the minimum level of significance.

\section{Results}

\section{Determining median lethal concentration doses of antihypertensives}

To determine the median lethal concentration $\left(\mathrm{LC}_{50}\right)$ of the antihypertensives, LDH release of hHeps, treated with different drug concentrations (dimethyl sulfoxide was used as solvent control) for 72 hours, was determined. $\mathrm{LC}_{50}$ values and resulting experimental doses of the drugs are summarized in Table 2.

\section{Antihypertensive drugs reduce ethanol- and rhTGF- $\beta_{1}$-dependent damage in hHeps}

We measured LDH activity in culture supernatants of hHeps treated with $100 \mathrm{mM}$ ethanol and/or $5 \mathrm{ng} / \mathrm{mL}$ rhTGF- $\beta_{1}$ in the presence or absence of antihypertensive drugs. After 72 hours both ethanol and rhTGF- $\beta_{1}$ induced cellular damage in hHeps with $50 \%-55 \%$ of total LDH released. Co-incubation with ethanol and TGF- $\beta_{1}$ enhanced the damage (Figure 1A). For ethanol, all drugs investigated significantly reduced the damaging effect in a dose-dependent manner (Figure 1B). Similar results were observed for rhTGF- $\beta_{1}$, except for furosemide, which had no effect on rhTGF- $\beta_{1}$-treated hHeps (Figure 1C). Interestingly, when hHeps were stimulated with ethanol and rhTGF- $\beta_{1}$ together, the protective effect of furosemide that was observed with ethanol alone was abolished (Figure 1D).

Table I Primer and polymerase chain reaction conditions

\begin{tabular}{|c|c|c|c|c|c|}
\hline Gene & GeneBank accession (NM_) & Forward primer $5^{\prime}-3^{\prime}$ & Reverse primer $5^{\prime}-3^{\prime}$ & $\mathbf{T}_{\mathrm{m}}\left({ }^{\circ} \mathrm{C}\right)$ & Product length (bp) \\
\hline \multirow[t]{2}{*}{ Smad2 } & 00100365 & CAAACCAGGTCTCT & GAGGCGGAAGT & 60 & 259 \\
\hline & & TGATGG & TCTGTTAGG & & \\
\hline \multirow[t]{2}{*}{ Smad3 } & 005902.2 & GGAGAAATGGTGC & GAAGGCGAACT & 60 & 258 \\
\hline & & GAGAAGG & CACACAGC & & \\
\hline \multirow[t]{2}{*}{ Smad4 } & 005359.3 & TGAATCCATATCAC & CAGGCTGACTT & 60 & 294 \\
\hline & & TACGAAC & GTGGAAG & & \\
\hline \multirow[t]{2}{*}{ Smad7 } & 005904.1 & TTCGGACAACAAG & AAGCCTTGATG & 60 & 201 \\
\hline & & AGTCAGC & GAGAAACC & & \\
\hline \multirow[t]{2}{*}{ Alk5 } & $0046 \mid 2.1$ & TGTTGGTACCCAAG & AACATCGTCGA & 60 & 287 \\
\hline & & GAAAGC & GCAATTTCC & & \\
\hline \multirow[t]{2}{*}{ GAPDH } & 007393 & CACCCACACTGTGC & СTCCTGCTTGC & 60 & 607 \\
\hline & & CCATC & TGATCCAC & & \\
\hline
\end{tabular}


Table $2 L_{50}$ and working concentrations of antihypertensives

\begin{tabular}{lll}
\hline Antihypertensive & LC $_{50}{ }^{*}$ & $\begin{array}{l}\text { Working } \\
\text { concentrations }\end{array}$ \\
\hline Amlodipine & $\mathrm{I} 1.73 \pm \mathrm{I} .06 \mu \mathrm{M}$ & $\mathrm{I} / 2 / 4 \mu \mathrm{M}$ \\
Captopril & $>100 \mu \mathrm{M}^{\circ}$ & $25 / 50 / 100 \mu \mathrm{M}$ \\
Furosemide & $36.73 \pm \mathrm{I} .1 \mathrm{I} \mu \mathrm{M}$ & $5 / 10 / 20 \mu \mathrm{M}$ \\
Metoprolol & $96.45 \pm 4.7 \mathrm{M}$ & $12.5 / 25 / 50 \mu \mathrm{M}$ \\
Propanolol & $31.47 \pm 1.37 \mu \mathrm{M}$ & $5 / 10 / 20 \mu \mathrm{M}$ \\
Spironolactone & $>100 \mu \mathrm{M}^{\circ}$ & $25 / 50 / 100 \mu \mathrm{M}$ \\
\hline
\end{tabular}

Notes: ${ }^{*} \mathrm{~N}=3, \mathrm{n}=4$; ${ }^{\circ}$ not in the range tested.

Abbreviation: $\mathrm{LC}_{50}$, median lethal concentration.

\section{Antihypertensives reduce ethanol- and rhTGF- $\beta_{1}$-dependent oxidative} stress in hHeps

We measured the formation of ROS in hHeps that were treated for 4 hours with $100 \mathrm{mM}$ ethanol and/or $5 \mathrm{ng} / \mathrm{mL}$ rhTGF- $\beta_{1}$ in the presence or absence of antihypertensives. As expected from previous investigations, ethanol and rhTGF- $\beta_{1}$ induced ROS formation in hHeps. Co-incubation with both substances together further increased ROS formation (Figure $2 \mathrm{~A}$ ). Although all drugs tested reduced ethanol- and rhTGF- $\beta_{1}$-dependent ROS formation (Figure $2 \mathrm{~B}-\mathrm{D}$ ), furosemide and spironolactone had the least effect for rhTGF- $\beta_{1}$ (Figure 2C).

\section{Antihypertensives rescue ethanol- and rhTGF- $\beta_{1}$-depleted glutathione levels in hHeps}

We measured glutathione (GSH) levels in hHeps that were treated for 4 hours with $100 \mathrm{mM}$ ethanol and/or $5 \mathrm{ng} / \mathrm{mL}$ rhTGF- $\beta_{1}$ in the presence or absence of antihypertensives. As expected from previous investigations, ethanol and rhTGF- $\beta_{1}$ reduced cellular GSH levels in hHeps. Co-incubation with both substances further decreased cellular GSH levels
A

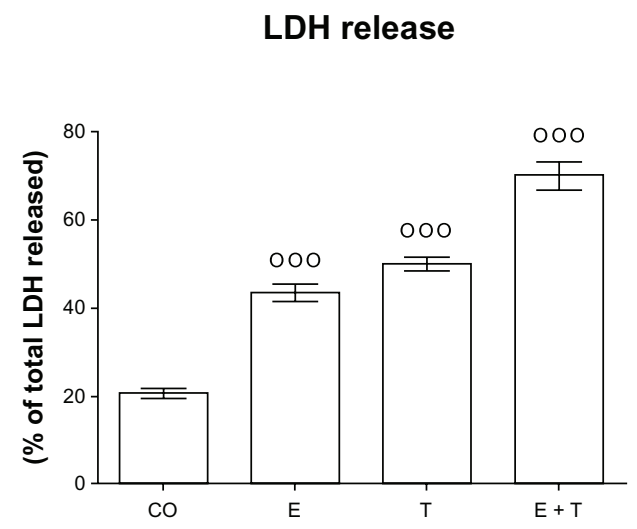

C

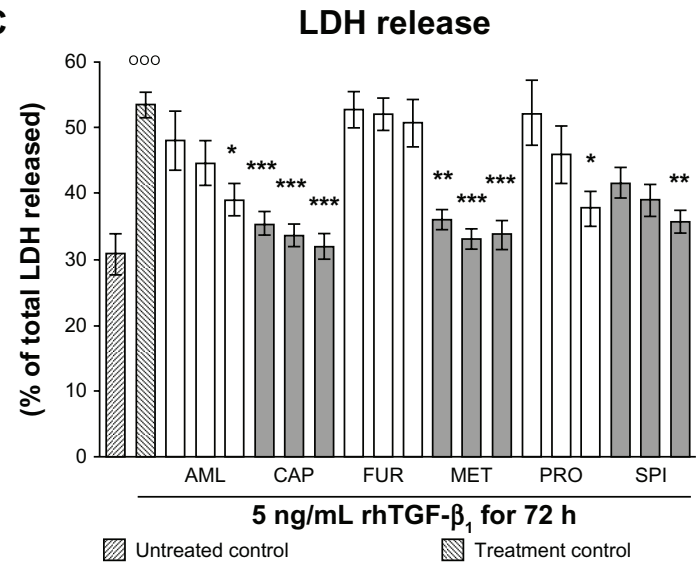

B

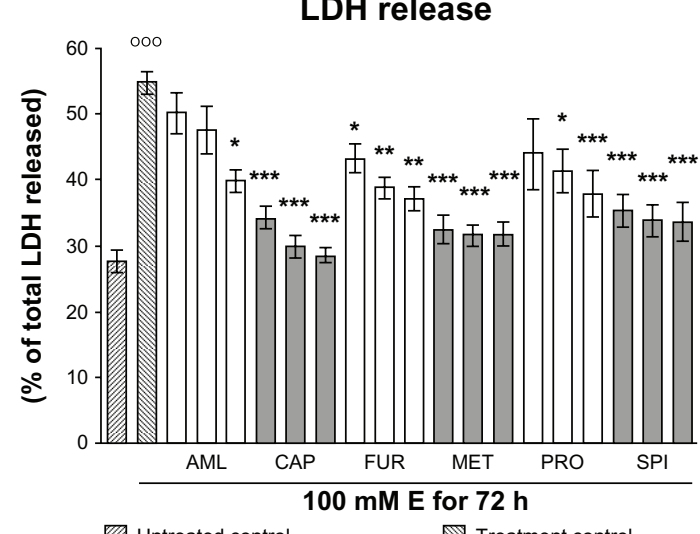

D

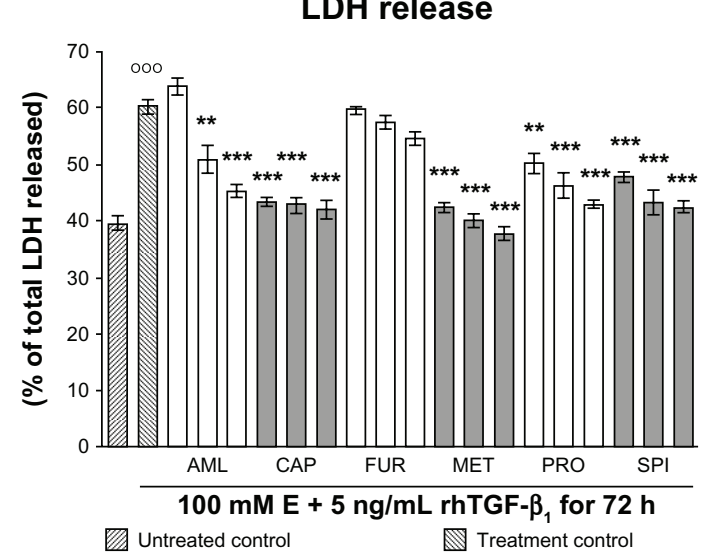

Figure I Effect of antihypertensives on E- and rhTGF- $\beta_{1}$-dependent cellular damage in hHeps. (A) LDH release of hHeps (N=4, $\left.\mathrm{n}=4\right)$ treated for 72 hours with I00 mM E and/or $5 \mathrm{ng} / \mathrm{mL}$ rhTGF- $\beta_{1}$. LDH release of hHeps $(\mathrm{N} \geq 3, \mathrm{n}=4)$ treated with (B) $100 \mathrm{mM} \mathrm{E},(\mathbf{C}) 5 \mathrm{ng} / \mathrm{mL}$ rhTGF- $\beta$, or (D) both substances for 72 hours in the presence or absence (treatment control) of AML (I/2/4 $\mu \mathrm{M})$, CAP (25/50/I00 $\mu \mathrm{M})$, FUR (5/I0/20 $\mu \mathrm{M})$, MET (I2.5/25/50 $\mu \mathrm{M})$, PRO (5/I0/20 $\mu \mathrm{M})$, or SPI (I2.5/25/50 $\mu \mathrm{M})$.

Notes: DMSO was used as solvent control. Results are given as percentage of total LDH released into the culture supernatant (cells dissolved with $0.1 \%$ Triton $\mathrm{X}$ - I00 equals $100 \%$ ). Basal toxicity (untreated control) was approximately $30 \% .{ }^{\circ 00} \mathrm{P}<0.00 \mathrm{I}$ as compared to untreated cells; $* \mathrm{P}<0.05$; $* * \mathrm{P}<0.0 \mathrm{I}$; $* * * P<0.00 \mathrm{I}$ as compared to $\mathrm{E}-\mathrm{or}^{*}$ rhTGF- $\beta$,-treated cells. Data are presented as mean \pm SEM.

Abbreviations: AML, amlodipine; CAP, captopril; DMSO, dimethyl sulfoxide; E, ethanol; FUR, furosemide; hHeps, human hepatocytes; LDH, lactate dehydrogenase; MET, metoprolol; PRO, propranolol; rhTGF- $\beta$ I, recombinant human transforming growth factor beta I; SPI, spironolactone. 
A

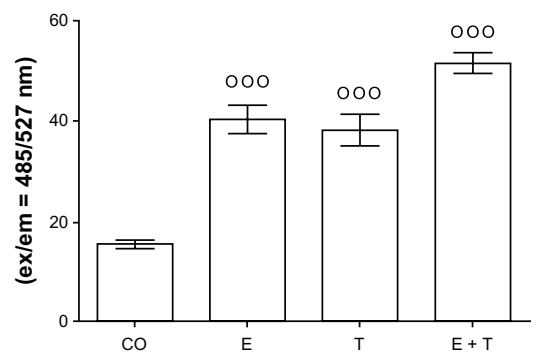

C

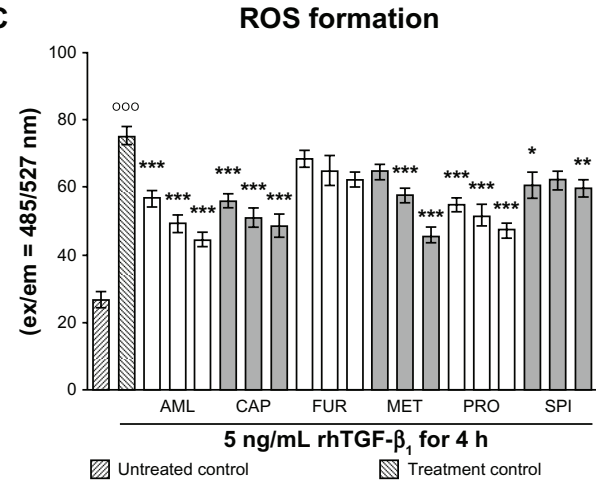

B

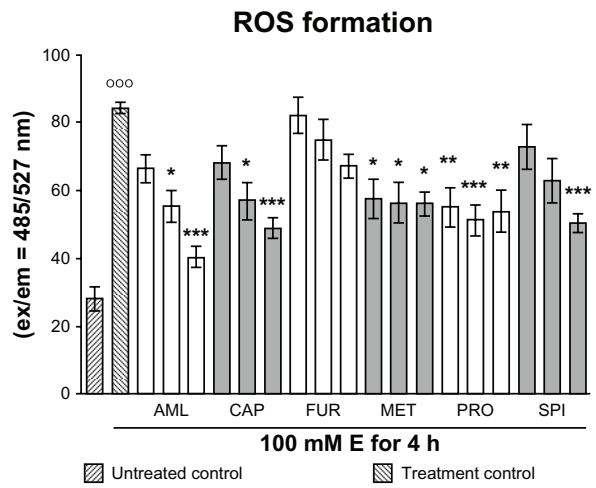

D

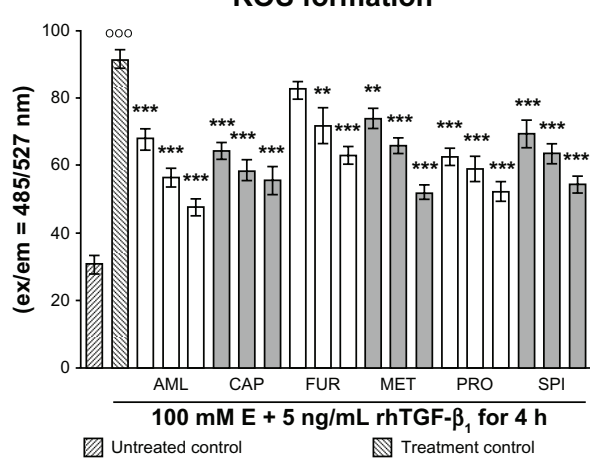

Figure 2 Effect of antihypertensives on $E$ and rhTGF- $\beta_{1}$-dependent ROS formation in hHeps. (A) ROS formation in hHeps ( $\mathrm{N}=3$; $\mathrm{n}=4$ ) treated with I00 mM E and/or $5 \mathrm{ng} / \mathrm{mL}$ rhTGF- $\beta$, for 4 hours. ROS measurement in hHeps $(\mathrm{N}=3, \mathrm{n}=4)$ treated with (B) $100 \mathrm{mM} \mathrm{E}$, (C) $5 \mathrm{ng} / \mathrm{mL}$ rhTGF- $\beta$, or $(\mathbf{D})$ both substances for 4 hours in the presence or absence (treatment control) of AML (I/2/4 $\mu \mathrm{M})$, CAP (25/50/I00 $\mu \mathrm{M})$, FUR (5/I0/20 $\mu \mathrm{M})$, MET (I2.5/25/50 $\mu \mathrm{M})$, PRO (5/I0/20 $\mu \mathrm{M})$, or SPI (I2.5/25/50 $\mu \mathrm{M})$. Data are presented as mean \pm SEM.

Notes: ${ }^{000} \mathrm{P}<0.00 \mathrm{I}$ as compared to untreated cells. DMSO was used as solvent control. Results are given as fluorescent intensities (ex/em $\left.=485 / 527 \mathrm{~nm}\right)$. Basal ROS formation (untreated control) was approximately 25 ; $* P<0.05$; **P $<0.0$ I; ***P $<0.00$ I as compared to E- or rhTGF- $\beta_{1}$-treated cells.

Abbreviations: AML, amlodipine; CAP, captopril; CO, control (untreated cells); E, ethanol; ex/em, excitation/emission; FUR, furosemide; hHeps, human hepatocytes; MET, metoprolol; PRO, propranolol; rhTGF- $\beta_{1}$, recombinant human transforming growth factor beta I; ROS, reactive oxygen species; SPI, spironolactone; T, treated with rhTGF- $\beta$ I.

(Figure 3A). Almost all drugs tested increased GSH levels reduced by ethanol and rhTGF- $\beta_{1}$ treatment dose dependently (Figure 3B-D). However, furosemide had the least effect for for both substances (Figure 3C).

\section{Antihypertensives induce $\mathrm{HO}-\mathrm{I}$ expression in hHeps}

We determined HO-1 expression levels in hHeps treated with the antihypertensives for 24 hours. For controls, we treated the cells with $100 \mathrm{mM}$ ethanol and/or $5 \mathrm{ng} / \mathrm{mL}$ rhTGF- $\beta_{1}$. Densitometric analysis revealed that all antihypertensives induced the expression of HO-1 in hHeps and showed strong effects for captopril, furosemide, and metoprolol (Figure 4A).

\section{Blocking HO-I activity abolished the protective effect of antihypertensives on ethanol- and rhTGF- $\beta_{1}$-dependent hepatocyte damage}

We measured LDH-release of hHeps treated with $100 \mathrm{mM}$ ethanol and/or $5 \mathrm{ng} / \mathrm{mL}$ rhTGF- $\beta_{1}$ in the presence or absence of antihypertensives. In order to block HO-1 activity, cells were co-incubated with $10 \mu \mathrm{M}$ zinc-protoporphyrin (ZnPP)-9. ZnPP9 completely abolished the beneficial effect of antihypertensives on ethanol-dependent hHeps damage (Figure 3B). For rhTGF- $\beta_{1}$-dependent damage where furosemide was not effective, ZnPP9 did not completely reverse the protective effects of the drugs (Figure 4C and D).

\section{Antihypertensives interfere with rhTGF- $\beta_{1}$-induced Smad3/4 signaling}

Primary hHeps were infected with Ad5-CAGA 9 -MLP-Luc adenoviral particles (Smad3/4-reporter construct) and stimulated for 24 hours with $5 \mathrm{ng} / \mathrm{mL}$ rhTGF- $\beta_{1}$ in the presence or absence of antihypertensives. Luciferase activity was measured in cell lysates. Interestingly, all drugs except furosemide (tendency for induction) were able to inhibit Smad3/4 signaling induced by rhTGF- $\beta_{1}$ (Figure $5 \mathrm{~A}$ ). To confirm that the interference was not at the receptor level, we repeated the experiment with hHeps co-infected with Ad5$\mathrm{CAGA}_{9}$-MLP-Luc adenoviral particles and viral particles encoding for a constitutive active TGF- $\beta$ receptor type I 
A

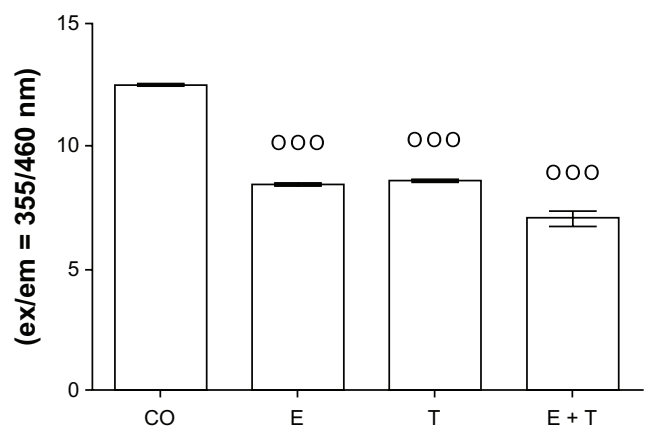

C

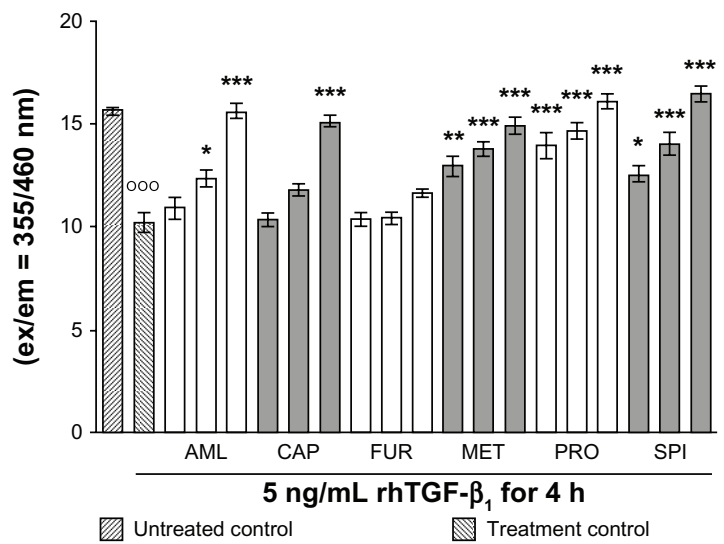

B

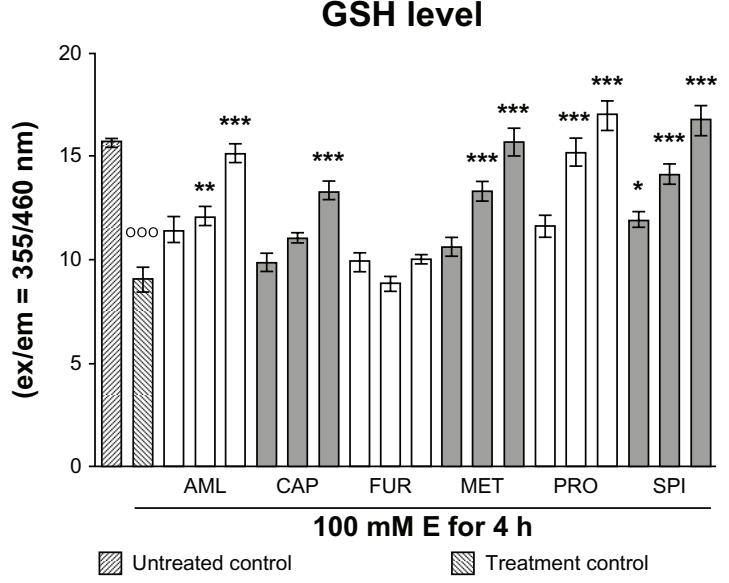

D

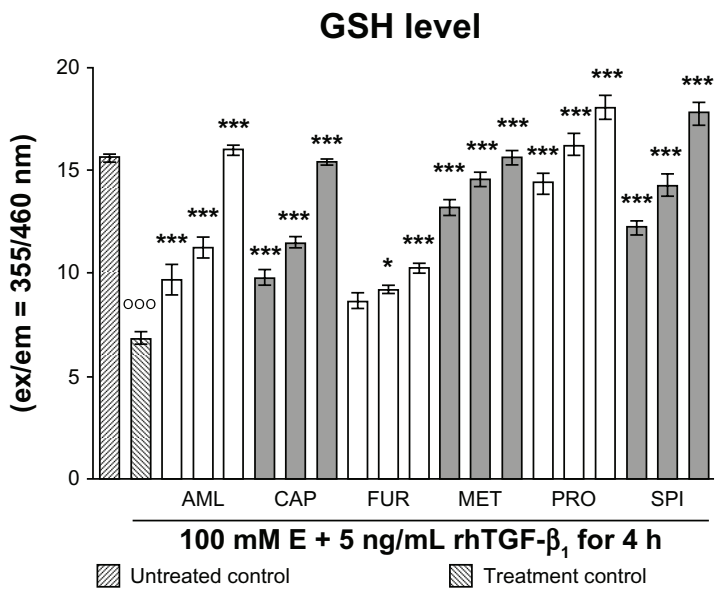

Figure 3 Effect of antihypertensives on cellular GSH levels in E- and rhTGF- $\beta_{1}$-treated hHeps. (A) GSH levels of hHeps $(\mathrm{N}=4$; $\mathrm{n}=4)$ treated with I00 mM E and/or $5 \mathrm{ng} / \mathrm{mL}$ rhTGF- $\beta$, for 4 hours. GSH levels of hHeps $(\mathrm{N}=4, \mathrm{n}=4)$ treated with (B) $100 \mathrm{mM} \mathrm{E},(\mathbf{C}) 5 \mathrm{ng} / \mathrm{mL}$ rhTGF- $\beta$, or (D) both substances for 4 hours in the presence or absence (treatment control) of AML (I/2/4 $\mu \mathrm{M})$, CAP (25/50/I00 $\mu \mathrm{M})$, FUR (5/I0/20 $\mu \mathrm{M})$, MET (I2.5/25/50 $\mu \mathrm{M})$, PRO (5/I0/20 $\mu \mathrm{M})$, or SPI (I2.5/25/50 $\mu \mathrm{M})$. Data are presented as mean \pm SEM.

Notes: DMSO was used as solvent control. Results are given as fluorescent intensities (ex/em = 355/460 nm). Basal GSH levels (untreated control) were approximately 16 . ${ }^{\circ 00} \mathrm{P}<0.00$ I as compared to untreated cells; $* \mathrm{P}<0.05$; $* * \mathrm{P}<0.0 \mathrm{I}$; ***P $<0.00$ I as compared to E- or rhTGF- $\beta$-treated cells.

Abbreviations: AML, amlodipine; CAP, captopril; CO, control (untreated cells); DMSO, dimethyl sulfoxide; E, ethanol; ex/em, excitation/emission; FUR, furosemide; GHS, glutathione; hHeps, human hepatocytes; MET, metoprolol; PRO, propranolol; rhTGF- $\beta_{1}$, recombinant human transforming growth factor beta I; SPI, spironolactone; $\mathrm{T}$, treated with rhTGF- $\beta$ I.

(Alk5-receptor) (Ad5-caAlk5), which does not require ligand binding for activating Smad3/4 signaling. For amlodipine, captopril, propranolol, and spironolactone, the inhibiting effect in Smad3/4 signaling remained, whereas in this assay, metoprolol no longer inhibited Smad3/4 signaling (Figure 5B).

\section{Antihypertensives alter expression levels of Smad3 and Smad4 in hHeps}

We measured expression levels of Smad2, -3, -4, -7, and Alk5 in hHeps that were treated for 24 hours with antihypertensives. We used $\beta$-actin as the house-keeping gene (Figure 5C). While Smad2, Smad7, and Alk5 expression levels were not significantly altered, expression levels of Smad3 were reduced by all antihypertensives (most notably for captopril and metoprolol), except for furosemide, which in contrast showed a significant induction of $\operatorname{Smad} 3$ and Smad4.

\section{Discussion}

Although the liver is probably the organ with the best regenerative capacity in the human body, repeated damage, eg, from alcohol abuse, causes liver cells to produce excessive extracellular matrix, which eventually prevents regeneration. As patients suffering from ALD show increased levels of reactive products and lipid peroxidation, ${ }^{1}$ it is widely accepted that oxidative stress plays an important role in the pathogenesis of ethanol-induced cellular injury.

In ALD patients, metabolism of consumed alcohol in the liver rapidly increases ROS production. ${ }^{2}$ Similarly, in our in vitro experiments, ethanol stimulation rapidly and 
A

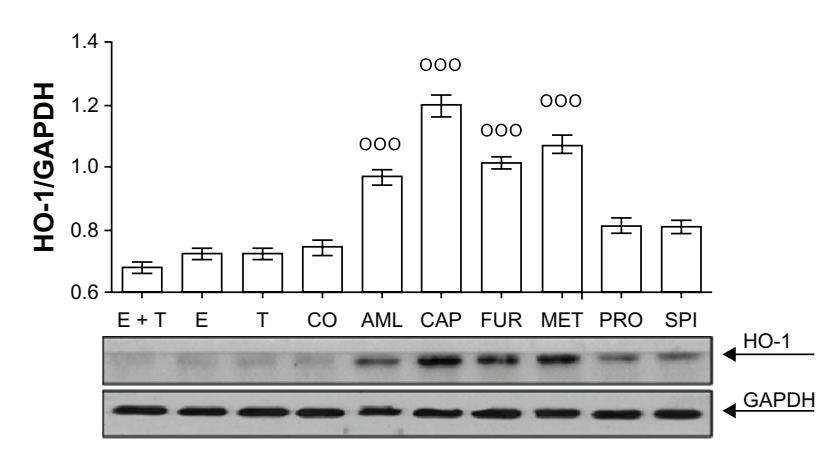

C

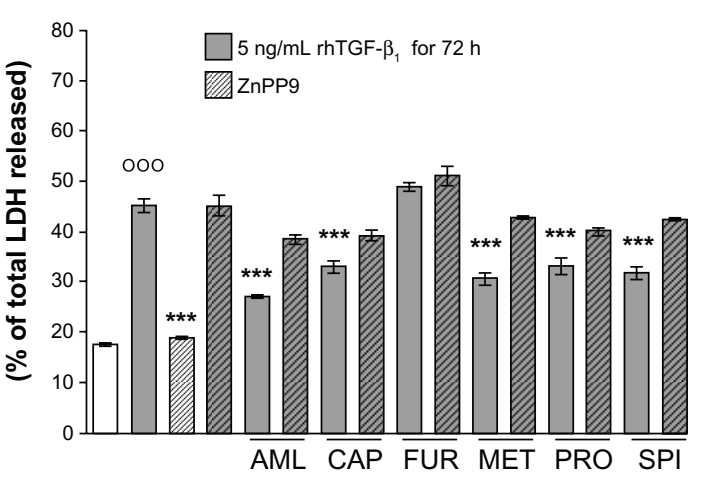

B

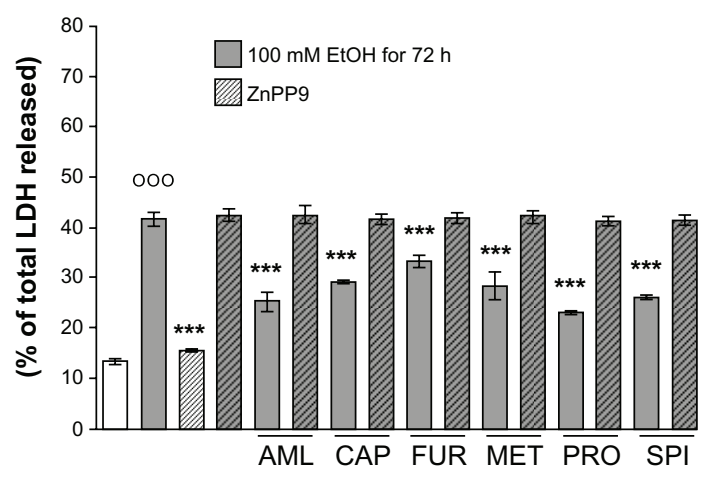

D

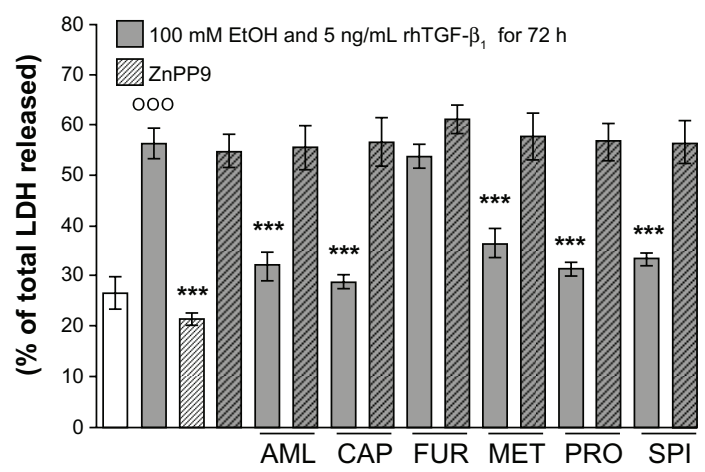

Figure 4 Effect of antihypertensives on HO-I expression in hHeps. (A) Representative Western blot picture of hHeps treated with I00 mM E and/or 5 ng/mL rhTGF- $\beta_{1}$ as well as $4 \mu \mathrm{M}$ AML, $100 \mu \mathrm{M}$ CAP, $20 \mu \mathrm{M}$ FUR, $50 \mu \mathrm{M}$ MET, $20 \mu \mathrm{M}$ PRO, or $50 \mu \mathrm{M}$ SPI for 24 hours. The membrane was probed for HO-I. GAPDH was used as loading control. LDH release of hHeps $(\mathrm{N}=3, \mathrm{n}=4)$ treated for 72 hours with $(\mathbf{B}) 100 \mathrm{mME}$ and/or $(\mathbf{C}$ and $\mathbf{D}) 5 \mathrm{ng} / \mathrm{mL}$ rhTGF- $\beta_{1}$ in the presence or absence of antihypertensives and $10 \mu \mathrm{M}$ of the HO-I inhibitor ZnPP9.

Notes: ${ }^{\circ 00} \mathrm{P}<0.00 \mathrm{I}$ as compared to untreated cells; ${ }^{*} P<0.05$; $* * P<0.0$ I; $* * * P<0.00 \mathrm{I}$ as compared to E- or rhTGF- $\beta$-treated cells. Data are presented as mean \pm SEM.

Abbreviations: AML, amlodipine; CAP, captopril; CO, control (untreated cells); E and EtOH, ethanol; FUR, furosemide; hHeps, human hepatocytes; HO-I, hemeoxigenase-I; LDH, lactate dehydrogenase; MET, metoprolol; PRO, propranolol; rhTGF- $\beta_{\text {I }}$, recombinant human transforming growth factor beta I; SPI, spironolactone; T, treated with rhTGF- $\beta$ I; ZnPP9, Zinc (II) Protoporphyrin 9.

dose-dependently induced ROS formation, which was even further enhanced by co-incubation with rhTGF- $\beta_{1}$. These results resemble our earlier observations in primary mouse hepatocytes. ${ }^{7}$ Increased formation of ROS in combination with reduced production of antioxidant enzymes and chemicals, particularly cellular glutathione, ${ }^{19}$ causes damage to hepatocytes. In our experiments approximately $40 \%-60 \%$ of the hHeps were damaged after 72 hours exposure to $100 \mathrm{mM}$ ethanol, with damage mainly due to apoptosis and not necrosis. ${ }^{3}$ In ALD patients, ethanol affects the immune system by altering cytokine production. ${ }^{6}$ Infiltrating macrophages in liver secrete profibrotic cytokines, eg, TGF$\beta_{1}$, whose signal transduction in the different liver cell types is critically required for chronic liver disease progression. TGF- $\beta_{1}$ activates the resident hepatic stellate cells to produce excessive extracellular matrix and even more TGF- $\beta_{1}$, which in turn further activates yet quiescent surrounding hepatic stellate cells. rhTGF- $\beta_{1}$ stimulates ROS production in rat hepatocytes by upregulating $\mathrm{NOX}^{8}$
In our in vitro experiments, exposure to rhTGF- $\beta_{1}$, similar to ethanol, dose dependently induced ROS formation. Interestingly, co-incubation of hHeps with ethanol and rhTGF- $\beta_{1}$, in contrast to murine hepatocytes in which pretreatment of the cells with ethanol sensitized them toward damage from rhTGF- $\beta_{1}$, did not significantly increase the sensitivity of the cells. ${ }^{7}$ However, this might be due to the higher basal toxicity levels in the hHeps.

We showed earlier that the calcium channel blockers nifedipine and verapamil protected hHeps from ethanoland rhTGF- $\beta_{1}$-dependent damage by upregulating the antioxidative enzyme HO- $1 .^{3}$ Today these calcium channel blockers are mainly used for the treatment of hypertensive crises. For a more general control of high blood pressure, a great variety of drugs are available, eg, novel calcium channel blockers, diuretics, ACE-inhibitors, aldosterone antagonists, renin inhibitors, angiotensin II receptor antagonists, adrenergic receptor agonists, or $\alpha$ - and $\beta$-blockers. Therefore, we tested the effect of the 
A

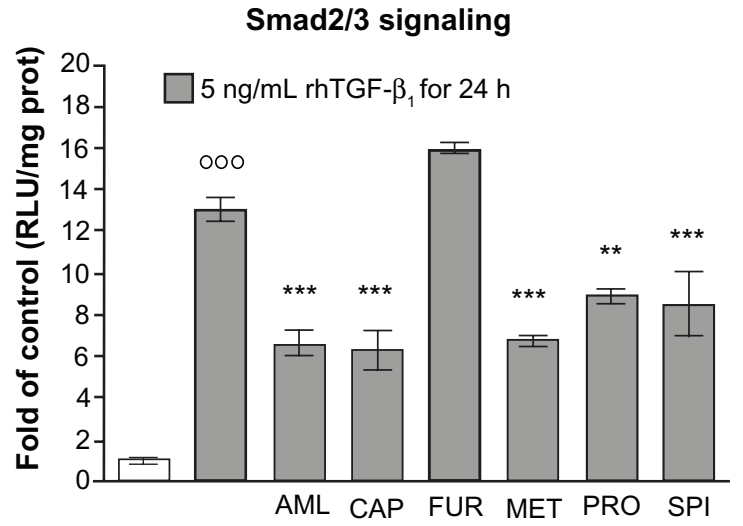

B

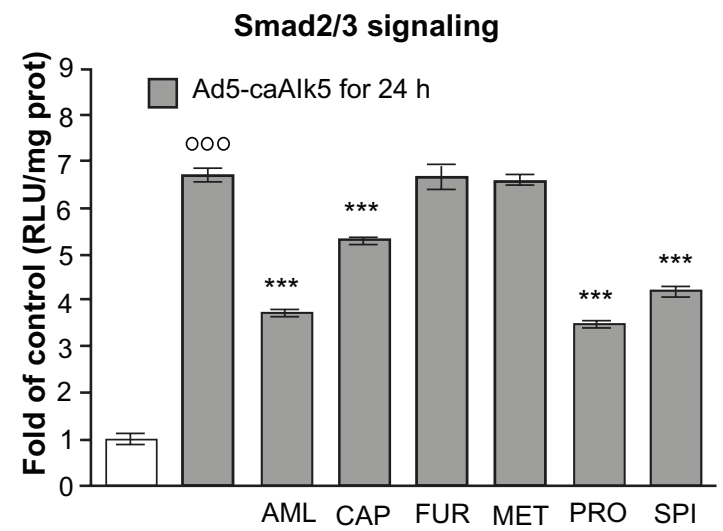

C

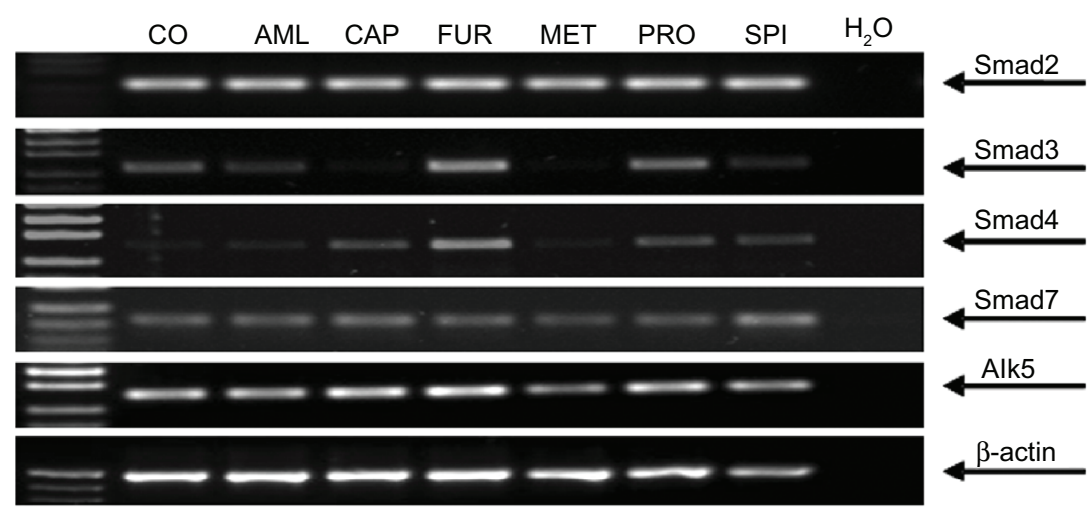

Figure 5 Effect of the antihypertensives on TGF- $\beta$ signaling. Adenoviral Smad3/4-reporter assay (Ad5-CAGA - MLP-Luc) of hHeps $(N=4, n=6)$ stimulated for 24 hours with/without antihypertensives and (A) $5 \mathrm{ng} / \mathrm{mL}$ rhTGF- $\beta_{1}$ or (B) co-infected with constitutive active Alk5. (C) Representative RT-PCR picture for Smad2, Smad3, Smad4, Smad7, and Alk5 of hHeps treated with $4 \mu$ M AML, $100 \mu$ M CAP, $20 \mu$ FUR, $50 \mu M$ MET, $20 \mu M$ PRO, or $50 \mu M$ SPI for 24 hours.

Notes: ${ }^{\circ 00} P<0.001$ as compared to untreated cells; $* P<0.05$; $* * P<0.01$; *** $P<0.00$ I as compared to rhTGF- $\beta_{1}$-treated cells. Data are presented as mean \pm SEM. Abbreviations: AML, amlodipine; CAP, captopril; CO, control (untreated cells); FUR, furosemide; hHeps, human hepatocytes; MET, metoprolol; PRO, propranolol; prot, protein; rhTGF- $\beta_{1}$, recombinant human transforming growth factor beta I; RLU, relative light unit; RT-PCR, reverse transcription polymerase chain reaction; SPI, spironolactone.

commonly used antihypertensives amlodipine, captopril, furosemide, metoprolol, propranolol, and spironolactone on ethanol- and rhTGF- $\beta_{1}$-treated hHeps. All substances reduced ethanol-induced ROS accumulation and the resulting cellular damage in a dose-dependent manner. The best protection was provided by captopril, metoprolol, and spironolactone, while a significant effect was only observed for amlodipine and propranolol at very high doses close to the $\mathrm{LC}_{50}$. Similarly, captopril, metoprolol, and spironolactone protected hHeps best from rhTGF- $\beta_{1}-$ induced cellular damage, while the effect of amlodipine and propranolol was strongly dose dependent. Results from the first reports on the in vivo effect of amlodipine and propranolol on myocardial and/or renal fibrosis varied from antifibrotic to noneffective, ${ }^{20-25}$ which might be a consequence of the respective applied doses. As a possible protective mechanism of amlodipine, a reduction in ROS by upregulating $\mathrm{Cu} / \mathrm{Zn}$ superoxide-dismutase was reported; ${ }^{26}$ captopril was also reported to reduce oxidative stress in vivo and thus to reduce myocardiac fibrosis. ${ }^{12,27}$

In the present experiments, all antihypertensives dose dependently reduced ethanol- or rhTGF- $\beta_{1}$-induced ROS formation. The protective effect observed when (poly)phenolic drugs were added to hHeps together with ethanol and/ or rhTGF- $\beta_{1}$ can be explained partly by the fact that (poly) phenolic substances due to their structure have strong free radical scavenging properties. As in the example of nifedipine and verapamil, they also are able to induce expression of the antioxidative enzyme $\mathrm{HO}-1 .{ }^{3} \mathrm{HO}-1$ is of particular interest as it is upregulated during oxidative stress and helps to protect the liver against damage from several chemical compounds, such as acetaminophen, carbon tetrachloride, and heavy metals. ${ }^{28}$ Thus, induction of HO-1 by cobalt protoporphyrin or the less toxic natural polyphenols quercetin and diallyldisulfide protect hHeps from ethanol-induced cytotoxicity. ${ }^{9-11}$ Captopril, furosemide and metoprolol were the strongest inducers of the antioxidative enzyme HO-1. Inhibition of 
HO-1 activity by ZnPP9 partly reversed the hepatocyte damage inhibitory effect of the drugs, identifying HO- 1 as a key downstream player of antihypertensives in mediating tissue protection. However, in contrast to ethanol, where furosemide significantly reduced cellular damage, rhTGF- $\beta_{1}$-dependent cellular damage was not affected by this drug. This suggests that besides antioxidative action, another mechanism is involved in the protective effect of the drugs.

In primary mouse hepatocytes, we found that furosemide reduces expression of alcohol-dehydrogenase 1 (data not shown), which has been described to negatively influence hepatocyte survival to these stimuli in mouse hepatocytes.? TGF- $\beta_{1}$-dependent Smad3/4 signaling is crucial for progression of both renal and hepatic fibrosis. Besides furosemide, all drugs tested significantly inhibited rhTGF$\beta_{1}$-dependent Smad3/4 signaling. The best inhibition of profibrotic Smad3/4 signaling was observed with amlodipine, captopril, and metoprolol, followed closely by spironolactone and propranolol. Furosemide by trend even enhanced Smad3/4 signaling. These results are supported by several in vitro and in vivo findings that showed reduced TGF- $\beta_{1}$ expression and activity upon treatment with these antihypertensives, mostly in renal cells. ${ }^{21,29-32}$ For example, amlodipine reduced adriamycin-induced toxicity in rat mesangial cells by affecting the expression of TGF- $\beta_{1}$, Smad2, and Smad4. ${ }^{31}$ Furthermore, captopril effectively decreased plasma levels of TGF- $\beta_{1}$, proteinuria, and uric acid in hypertensive kidney transplant patients. ${ }^{32}$ In addition, captopril limited TGF- $\beta_{1}$ over-expression in acute normotensive anti-Thy 1 glumerulonephritis..$^{30}$ In patients with chronic kidney disease, spironolactone effectively reduced renal fibrosis and urinary TGF- $\beta_{1}$ excretion, emphasizing the link between both parameters; ${ }^{29}$ similarly, it may prevent renal dysfunction and fibrosis induced by tacrolimus. ${ }^{32}$ Interestingly, metoprolol does not display antifibrotic effects in transgenic mice with TGF- $\beta_{1}$-induced fibrosis. ${ }^{33}$

To more closely investigate the Smad3/4-inhibitory mechanism of the drugs, we co-infected the cells with an adenovirus that expresses a constitutively active Alk5-receptor, which induces Smad3/4 signaling independent of ligand binding to the receptors. In this setup, metoprolol could no longer inhibit Smad3/4 signaling, suggesting an interference of the drug with ligand/receptor binding. Interestingly, Alk5 was reduced by metoprolol at the mRNA level, but none of the other drugs that were investigated. Smad2 and Smad7 expression levels were not significantly affected by any of the antihypertensives. However, Smad 3 and Smad4 expression levels were strongly reduced, especially in hHeps treated with amlodipine, captopril, metoprolol, propranolol, and spironolactone. Smad3 and Smad4 expression levels were notably increased with furosemide, which may be an explanation for the enhanced signaling observed.

Our data suggest that high blood pressure treatment with even low doses of captopril, metoprolol, or spironolactone may additionally have beneficial effects on liver fibrosis in patients with ALD. Although amlodipine and propranolol also reduced ethanol-and rhTGF- $\beta_{1}$-dependent cellular damage, the effect was strongly dose dependent and present only at higher concentrations, exacerbating the choice of the therapeutic dose. This is supported by animal studies effectively using propranolol to delay progression of sclerosing cholangitis in multi-drug resistance protein 2 (MDR2)-knockout mice. ${ }^{16}$ Furthermore, captopril but not amlodipine was shown to reduce fibrotic markers in experimental nonalcoholic steatohepatitis (NASH) ${ }^{17}$ or after bile-duct ligation. ${ }^{15}$ However, captopril is also reported to directly interfere with matrix metalloproteinase activity, thus inhibiting the degradation of extracellular matrix and further favoring fibrotic tissue changes.$^{13}$ On the other hand, captopril may induce upregulation of metallopeptidase with thrombospondin type 1 (ADAMTS-1), which is known to accelerate the degradation of type I collagen, ${ }^{34}$ which would compensate for the above. Interestingly, furosemide protected hHeps only against ethanol-induced damage but not against rhTGF- $\beta_{1}$-induced damage. We could show that despite an induction of HO- 1 , furosemide enhances profibrotic TGF- $\beta_{1}$ induced Smad3/4 signaling, suggesting that this drug might even favor liver fibrosis in vivo. Special care has to be taken when different antihypertensives are used in combination. Eplerenone, for example, is reported to potentiate the effect of amlodipine against cardiac inflammation and fibrosis in salt-sensitive hypertensive rats. ${ }^{35}$ Similarly, in a rat model of genetic hypertrophic cardiomyopathy, a combination therapy of low-dose spironolactone and captopril more effectively reduced fibrosis markers when compared with the single drugs. ${ }^{36}$

In summary, our data suggest that antihypertensives might influence progression of liver fibrosis by modulating HO-1 activity in liver cells. Increased HO-1 activity protects hHeps from ROS-dependent damage by increasing cellular GSH. Under these conditions GSH favors formation of nontoxic products from ROS. Although all drugs tested induced HO-1 expression in hHeps, protecting them from excessive ROS production, the effect of amlodipine and propranolol was only observed at high concentrations close to the $\mathrm{LC}_{50}$. Therefore, drugs, eg, captopril, metoprolol, or spironolactone, already 
protecting hHeps from ethanol- and rhTGF- $\beta_{1}$-induced damage at low concentrations, might be more suitable to reduce liver fibrosis in vivo. Furthermore, furosemide even induced profibrogenic TGF- $\beta_{1}$ signaling, suggesting that this drug might facilitate liver fibrosis in vivo. Therefore, the choice of antihypertensive used for reducing high blood pressure might be crucial in patients with ALD as they might, in the worst case, further promote liver fibrosis, while in the best case even reduce or prevent its progression. Thus, our in vitro data suggest antihypertensive therapy could be adjusted in order to support the antifibrotic therapy, which needs to be further explored in vivo.

\section{Acknowledgments}

This work was partially supported by the Federal Ministry of Research (BMBF - 0315208E; BMBF - 01GN0984).

\section{Author contributions}

Sabrina Ehnert, Teresa Lukoschek, and Andreas K Nussler designed the study. Sabrina Ehnert, Teresa Lukoschek, Anastasia Bachmann, and Juan José Martínez Sánchez carried out the research. Sabrina Ehnert, Terersa Lukoschek, and Sebastian Mueller analyzed the data. Natascha C Nussler, Stefan Pscherer, and Andreas K Nussler provided the material for the study. Sabrina Ehnert, Teresa Lukoschek, Anastasia Bachmann, and Andreas K Nussler wrote the paper. Anastasia Bachmann, Georg Damm, Stefan Pscherer, Steven Dooley, and Sebastian Mueller critically reviewed the paper.

\section{Disclosure}

The authors report no conflicts of interest in this work.

\section{References}

1. Farfan Labonne BE, Gutierrez M, Gomez-Quiroz LE, et al. Acetaldehydeinduced mitochondrial dysfunction sensitizes hepatocytes to oxidative damage. Cell Biol Toxicol. 2009;25:599-609.

2. Cederbaum AI, Lu Y, Wu D. Role of oxidative stress in alcohol-induced liver injury. Arch Toxicol. 2009;83:519-548.

3. Nussler AK, Hao L, Knobeloch D, et al. Protective role of HO-1 for alcohol-dependent liver damage. Dig Dis. 2010;28:792-798.

4. Lakshmi Devi S, Anuradha CV. Mitochondrial damage, cytotoxicity and apoptosis in iron-potentiated alcoholic liver fibrosis: amelioration by taurine. Amino Acids. 2010;38:869-879.

5. Sastre J, Serviddio G, Pereda J, et al. Mitochondrial function in liver disease. Front Biosci. 2007;12:1200-1209.

6. Albano E, Vidali M. Immune mechanisms in alcoholic liver disease. Genes Nutr. 2010;5:141-147.

7. Ciuclan L, Ehnert S, Ilkavets I, et al. TGF-beta enhances alcohol dependent hepatocyte damage via down-regulation of alcohol dehydrogenase I. J Hepatol. 2010;52:407-416.

8. Carmona-Cuenca I, Roncero C, Sancho P, et al. Upregulation of the NADPH oxidase NOX4 by TGF-beta in hepatocytes is required for its pro-apoptotic activity. J Hepatol. 2008;49:965-976.
9. Liu LG, Yan H, Zhang W, et al. Induction of heme oxygenase-1 in human hepatocytes to protect them from ethanol-induced cytotoxicity. Biomed Environ Sci. 2004;17:315-326.

10. Bao W, Li K, Rong S, et al. Curcumin alleviates ethanol-induced hepatocytes oxidative damage involving heme oxygenase-1 induction. J Ethnopharmacol. 2010;128:549-553.

11. Yao P, Nussler A, Liu L, et al. Quercetin protects human hepatocytes from ethanol-derived oxidative stress by inducing heme oxygenase-1 via the MAPK/Nrf2 pathways. J Hepatol. 2007;47:253-261.

12. Kumar S, Enjamoori R, Jaiswal A, et al. Catecholamine-induced myocardial fibrosis and oxidative stress is attenuated by Terminalia arjuna (Roxb). J Pharm Pharmacol. 2009;61:1529-1536.

13. Okada M, Kosaka N, Hoshino Y, et al. Effects of captopril and telmisartan on matrix metalloproteinase- 2 and -9 expressions and development of left ventricular fibrosis induced by isoprenaline in rats. Biol Pharm Bull. 2011;33:1517-1521.

14. Ardekani GS, Aghaei S, Nemati MH, et al. Treatment of a postburn keloid scar with topical captopril: report of the first case. Plast Reconstr Surg. 2009;123:112e-113e.

15. Kim MY, Baik SK, Park DH, et al. Angiotensin receptor blockers are superior to angiotensin-converting enzyme inhibitors in the suppression of hepatic fibrosis in a bile duct-ligated rat model. $J$ Gastroenterol. 2008;43:889-896.

16. Strack I, Schulte S, Varnholt H, et al. beta-Adrenoceptor blockade in sclerosing cholangitis of Mdr2 knockout mice: antifibrotic effects in a model of nonsinusoidal fibrosis. Lab Invest. 2011;91:252-261.

17. Toblli JE, Munoz MC, Cao G, et al. ACE inhibition and AT1 receptor blockade prevent fatty liver and fibrosis in obese Zucker rats. Obesity (Silver Spring). 2008;16:770-776.

18. Nussler A, Konig S, Ott M, et al. Present status and perspectives of cell-based therapies for liver diseases. J Hepatol. 2006;45: 144-159.

19. Garcia-Ruiz C, Fernandez-Checa JC. Mitochondrial glutathione: hepatocellular survival-death switch. J Gastroenterol Hepatol. 2006; 21 Suppl 3:S3-S6.

20. Fogari R, Mugellini A, Destro M, et al. Losartan and amlodipine on myocardial structure and function: a prospective, randomized, clinical trial. Diabet Med. 2011;29:24-31.

21. Ma G, Allen TJ, Cooper ME, et al. Calcium channel blockers, either amlodipine or mibefradil, ameliorate renal injury in experimental diabetes. Kidney Int. 2004;66:1090-1098.

22. Pacca SR, de Azevedo AP, De Oliveira CF, et al. Attenuation of hypertension, cardiomyocyte hypertrophy, and myocardial fibrosis by beta-adrenoceptor blockers in rats under long-term blockade of nitric oxide synthesis. J Cardiovasc Pharmacol. 2002;39:201-207.

23. Perlini S, Palladini G, Ferrero I, et al. Sympathectomy or doxazosin, but not propranolol, blunt myocardial interstitial fibrosis in pressureoverload hypertrophy. Hypertension. 2005;46:1213-1218.

24. Sevilla MA, Voces F, Carron R, et al. Amlodipine decreases fibrosis and cardiac hypertrophy in spontaneously hypertensive rats: persistent effects after withdrawal. Life Sci. 2004;75:881-891.

25. Shibasaki Y, Nishiue T, Masaki H, et al. Impact of the angiotensin II receptor antagonist, losartan, on myocardial fibrosis in patients with end-stage renal disease: assessment by ultrasonic integrated backscatter and biochemical markers. Hypertens Res. 2005;28: 787-795

26. Umemoto S, Tanaka M, Kawahara S, et al. Calcium antagonist reduces oxidative stress by upregulating $\mathrm{Cu} / \mathrm{Zn}$ superoxide dismutase in strokeprone spontaneously hypertensive rats. Hypertens Res. 2004;27: 877-885.

27. Simko F, Pechanova O, Pelouch V, et al. Effect of melatonin, captopril, spironolactone and simvastatin on blood pressure and left ventricular remodelling in spontaneously hypertensive rats. J Hypertens Suppl. 2009;27:S5-S10.

28. Farombi EO, Surh YJ. Heme oxygenase-1 as a potential therapeutic target for hepatoprotection. J Biochem Mol Biol. 2006;39: 479-491. 
29. Guney I, Selcuk NY, Altintepe L, et al. Antifibrotic effects of aldosterone receptor blocker (spironolactone) in patients with chronic kidney disease. Ren Fail. 2009;31:779-784.

30. Peters H, Ruckert M, Gaedeke J, et al. Angiotensin-converting enzyme inhibition but not beta-adrenergic blockade limits transforming growth factor-beta overexpression in acute normotensive antithy1 glomerulonephritis. J Hypertens. 2003;21:771-780.

31. Song YJ, Li J, Xie XF, et al. Effects of amlodipine on TGF-beta-induced Smad2, 4 expressions in adriamycin toxicity of rat mesangial cells. Arch Toxicol. 2011;85:663-668.

32. Shin GT, Kim SJ, Ma KA, et al. ACE inhibitors attenuate expression of renal transforming growth factor-betal in humans. Am J Kidney Dis. 2000;36:894-902.

33. Seeland U, Schaffer A, Selejan S, et al. Effects of AT1- and beta-adrenergic receptor antagonists on TGF-beta1-induced fibrosis in transgenic mice. Eur J Clin Invest. 2009;39:851-859.
34. Guo C, Wang Y, Liang H, et al. ADAMTS-1 contributes to the antifibrotic effect of captopril by accelerating the degradation of type I collagen in chronic viral myocarditis. Eur J Pharmacol. 2011;629: 104-110.

35. Nakamura T, Fukuda M, Kataoka K, et al. Eplerenone potentiates protective effects of amlodipine against cardiovascular injury in salt-sensitive hypertensive rats. Hypertens Res. 2011;34:817-824.

36. de Resende MM, Kriegel AJ, Greene AS. Combined effects of low-dose spironolactone and captopril therapy in a rat model of genetic hypertrophic cardiomyopathy. J Cardiovasc Pharmacol. 2006;48:265-273.

\section{Publish your work in this journal}

Hepatic Medicine: Evidence and Research is an international, peerreviewed, open access journal covering all aspects of adult and pediatric hepatology in the clinic and laboratory including the following topics: Pathology, pathophysiology of hepatic disease; Investigation and treatment of hepatic disease; Pharmacology of drugs used for

\section{Dovepress}

the treatment of hepatic disease. Issues of patient safety and quality of care will also be considered. The manuscript management system is completely online and includes a very quick and fair peer-review system, which is all easy to use. Visit http://www.dovepress.com/ testimonials.php to read real quotes from published authors.

Submit your manuscript here: http://www.dovepress.com/hepatic-medicine-evidence-and-research-journal 